journal club

\title{
Das Wohlbefinden lässt sich fördern
}

Fragestellung: Kann man durch Interventionen und Veränderungen im Lernumfeld der Medizinstudierenden deren Wohlbefinden verbessern?

Hintergrund: Viele Studien zeigten, dass Studierende der $\mathrm{Hu}-$ manmedizin ein erhöhtes Risiko haben, depressive Symptome bis hin zu Suizidgedanken zu entwickeln. Mittlerweile gibt es eine Vielzahl an Untersuchungen mit verschiedenen Interventionen, mit dem Ziel, die seelische Gesundheit von Medizinstudierenden zu verbessern. Dieser Review versucht einen Überblick über die Untersuchungen an US-amerikanischen Medical Schools zu schaffen, um erste Erkenntnisse vorzulegen, aber auch weitere Untersuchungen anzuregen.

Patienten und Methodik: Analysiert wurden 28 Studien, die als Outcome-Kriterien die Verbesserung des Wohlbefindens von Studenten der Humanmedizin durch Änderungen im Lernumfeld hatten. Die Teilnehmeranzahl betrug mindestens 8.224 (eine Studie gab keine Probandenanzahl an). Es flossen Studien mit unterschiedlichen Designs ein (zehn postinterventionelle Untersuchungen, zwei Interventionsstudien mit Vor- und Nachtestung, aber ohne Kontrollgruppe, 13 Studien mit nicht randomisierter und drei mit randomisierter Kontrollgruppe). Messinstrumente waren semi-

Wasson LT, Cusmano A, Meli L et al. Prevalence of depression, association between learning environment interventions and medical Student well-being. JAMA 2016; 316: 2237-52 strukturierte Interviews oder andere quantitative Verfahren. Die Interventionen zur Verbesserung des Wohlbefindens umfassten Veränderungen der Notenvergabe (Nominal- versus Ordinal- skala), vereinfachten Zugang zu Gesundheitsleistungen und Früherkennung, Mind-Body-Programme, Änderungen im Aufbau des Curriculums, Mentoring, längerfristige Zusammenschlüsse von Arbeitsgruppen und Anleitung zur Selbsthypnose. Bei großer methodischer Heterogenität wurden die verschiedenen Studien anhand des MERSQI (Medical Education Research Study Quality Instrument) evaluiert.

Ergebnisse: Die Erfolge der Interventionen waren sehr unterschiedlich. Studien, die Nominal- mit Ordinalskalen in Prüfungen (bestanden/durchgefallen versus Notensystem) verglichen, zeigten ein besseres Wohlbefinden bei Verwendung von Nominalskalen. Studien, die eine verbesserte Früherkennung psychischer Probleme und einen verbesserten Zugang zu Fürsorgestellen untersuchten, konnten ein verbessertes Wohlbefinden nicht signifikant bestätigen. Durch Body-Mind-Programme konnte eine Verbesserung von Selbstachtsamkeit und Stressreduktion festgestellt werden. Unterschiedliche Curricula hatten keinen Einfluss auf das Wohlbefinden der Studierenden. Studien, die mehrere Interventionen durchführten, kamen alle zu einer signifikanten Verbesserung, die eine Langzeitzusammenarbeit von Peers in einer festen Gruppe, Wellness-Programme, Mentoring und Veränderung der Notenvergabe beinhaltete. Interventionen zur Selbsthypnose waren nicht wirksam. Mentoring-Programme führten entweder zu subjektiven Verbesserungen der seelischen Stabilität oder zu keinen signifikanten Veränderungen.

Schlussfolgerungen: Trotz vieler Limitationen zeigt sich, dass es Interventionen gibt, die das Wohlbefinden von Medizinstudierenden fördern (keine Noten im Studium, Body-Mind-Programme, Mentoring).

\section{- Kommentar von Stefan Schenkel und Nadine Dreimüller, Mainz}

\section{Gesund durch das Studium}

In der Zusammenschau lässt sich nach Betrachtung der einzelnen Studien keine überzeugende Evidenz und weitere Empfehlung für spezifische Interventionen ableiten. Auch aufgrund der eher mäßigen Qualität der Studien lassen sich keine eindeutigen Schlüsse ziehen. Die methodischen Defizite der Studien waren vielfältig (fehlende oder nicht randomisierte Kontrollgruppen, fehlende Definition des "Wohlbefindens" und ähnliches). Aber um nachhaltige Veränderungen zu bewirken bedarf es mehr hochwertiger Studien. Trotz aller Limitationen zeigten verschiedene Interventionen eine Verbesserung des Wohlbefindens. Da internationale Studien deutlich zeigen konnten, dass gerade Studierende der Humanmedizin ein erhöhtes Risiko haben, im Verlauf des Studiums psychisch zu erkranken, sind präventive Maßnahmen und entsprechende Interventionen von hoher Bedeutung. Durch die Fokussierung dieses Reviews auf das US-amerikanische Ausbildungssystem ist keine völlige Übertragbarkeit auf das deutsche
Universitätssystem möglich. Für Deutschland liegen bislang nur sehr wenige Studien zu Interventionen vor. Dieses Review kann als Ausgangspunkt dafür dienen, welche Interventionen zur Verbesserung der psychischen Gesundheit auch in Deutschland sinnvoll getestet werden sollten.

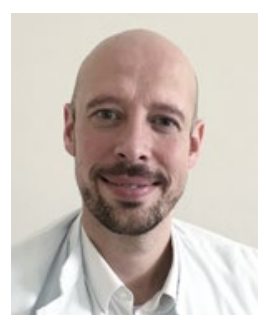

Dr. med. Stefan Schenkel, Mainz

Klinik für Psychiatrie und Psychotherapie, Universitätsmedizin Mainz E-Mail:Stefan.Schenkel@unimedizin-mainz.de 